The Making of Rehabilitation 
This page intentionally left blank 


\section{The Making of Rehabilitation A Political Economy of Medical Specialization, 1890-1980}

\section{Glenn Gritzer}

and Arnold Arluke

UNIVERSITY OF CALIFORNIA PRESS

Berkeley - Los Angeles - London 
University of California Press

Berkeley and Los Angeles, California

University of California Press, Ltd.

London, England

Copyright (C) 1985 by The Regents of the University of California

Library of Congress Cataloging in Publication Data

Gritzer, Glenn.

The making of rehabilitation medicine.

(Comparative studies of health systems and medical care)

Includes index.

1. Rehabilitation-Political aspects-History-

19th century. 2. Rehabilitation-Political aspectsUnited States-History-19th century. 3. Rehabilitation -Political aspects-History-20th century. 4. Rehabilitation-Political aspects--United States-History20th century. 5. Rehabilitation-Economic aspectsHistory-19th century. 6. Rehabilitation-Economic aspects-United States-History-19th century.

7. Rehabilitation-Economic aspects-History-20th century. 8. Rehabilitation-Economic aspects--

United States-History-20th century. I. Arluke, Arnold. II. Title. III. Series. [DNLM: 1. Occupational Therapy -history-United States. 2. Physical Medicine-history -United States. 3. Physical Therapy-historyUnited States. 4. Rehabilitation-history-United States. WZ 70 AA1 G68m]

$\begin{array}{lccc}\text { RM930.G75 } & 1985 & 362.1^{\prime} 7866^{\prime} 0973 & 84-28008\end{array}$

ISBN 0-520-06604-9

Printed in the United States of America

$\begin{array}{llllllll}08 & 07 & 06 & 05 & 04 & 03 & 02 & 01\end{array}$

$\begin{array}{lllllll}9 & 8 & 7 & 6 & 5 & 4 & 3\end{array}$

The paper used in this publication meets the minimum requirements of ANSI/NISO Z39.48-1992 (R 1997) (Permanence of Paper). () 
To our parents 
This page intentionally left blank 